\title{
Interactions between Liquid-Wall Vapor and Edge Plasmas
}

\author{
T.D. Rognlien, M.E. Rensink
}

This article was submitted to $14^{\text {th }}$ International Conference on Plasma Surface Interactions in Controlled Fusion Devices Rosenheim, Germany, May 22-26, 2000

\section{May 25, 2000}

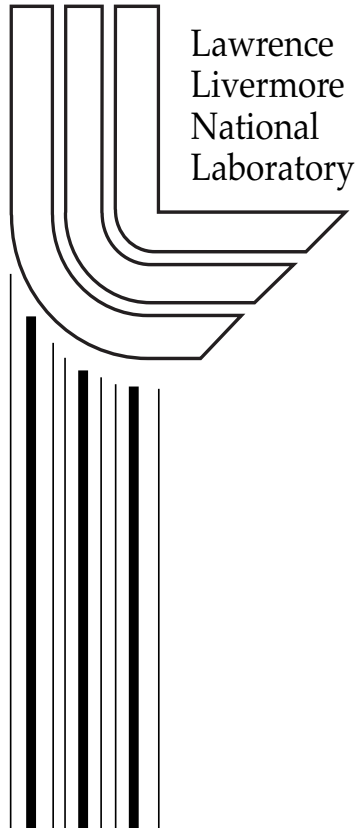




\section{DISCLAIMER}

This document was prepared as an account of work sponsored by an agency of the United States Government. Neither the United States Government nor the University of California nor any of their employees, makes any warranty, express or implied, or assumes any legal liability or responsibility for the accuracy, completeness, or usefulness of any information, apparatus, product, or process disclosed, or represents that its use would not infringe privately owned rights. Reference herein to any specific commercial product, process, or service by trade name, trademark, manufacturer, or otherwise, does not necessarily constitute or imply its endorsement, recommendation, or favoring by the United States Government or the University of California. The views and opinions of authors expressed herein do not necessarily state or reflect those of the United States Government or the University of California, and shall not be used for advertising or product endorsement purposes.

This is a preprint of a paper intended for publication in a journal or proceedings. Since changes may be made before publication, this preprint is made available with the understanding that it will not be cited or reproduced without the permission of the author.

This report has been reproduced directly from the best available copy.

Available electronically at http://www.doc.gov/bridge

Available for a processing fee to U.S. Department of Energy

And its contractors in paper from

U.S. Department of Energy

Office of Scientific and Technical Information

P.O. Box 62

Oak Ridge, TN 37831-0062

Telephone: (865) 576-8401

Facsimile: (865) 576-5728

E-mail: reports@adonis.osti.gov

Available for the sale to the public from

U.S. Department of Commerce

National Technical Information Service

5285 Port Royal Road

Springfield, VA 22161

Telephone: (800) 553-6847

Facsimile: (703) 605-6900

E-mail: orders@ntis.fedworld.gov

Online ordering: http:/ / www.ntis.gov/ordering.htm

\section{OR}

Lawrence Livermore National Laboratory

Technical Information Department's Digital Library

http: / / www.llnl.gov/tid/Library.html 


\title{
Interactions between Liquid-Wall Vapor and Edge Plasmas
}

\author{
T.D. Rognlien and M.E. Rensink \\ Lawrence Livermore National Laboratory, \\ Livermore, California 94551 USA
}

The use of liquid walls for fusion reactors could help solve problems associated with material erosion from high plasma heat-loads and neutronic activation of structures. A key issue analyzed here is the influx of impurity ions to the core plasma from the vapor of liquid side-walls. Numerical 2D transport simulations are performed for a slab geometry which approximates the edge region of a reactor-size tokamak. Both lithium vapor (from $\mathrm{Li}$ or $\mathrm{SnLi}$ walls) and fluorine vapor (from Flibe walls) are considered for hydrogen edge-plasmas in the high- and low-recycling regimes. It is found that the minimum influx is from lithium with a low-recycling hydrogen plasma $\Gamma$ and the maximum influx occurs for fluorine with a high-recycling hydrogen plasma. 


\section{Introduction}

The use of flowing liquid surfaces as first walls and divertor plates for fusion reactors has a number of attractive features; these include continuous replenishment $\Gamma$ possible high heat-load capabilities Tand elimination of solid structures that become activated by neutrons (e.g. Tsee Refs. 12). The operation of tokamak devices in the presence of various impurity species has been investigated experimentally on various tokamaks. For examplerthe impact of lithium and its role in recycling for the TFTR tokamak is reported in Ref. 35while the use of neon and argon in the DIII-D tokamak is described in Ref. 4.

A major concern for the use of liquid walls in fusion reactors is contamination of the core by impurities. The acceptable core impurity level is determined by dilution and radiation; for impurities with low and moderate nuclear charge $\Gamma Z \Gamma$ dilution is the dominant constraint. If one assumes equal core densities of deuterium and tritium $\Gamma$ $n_{D}=n_{h} / 2$ and $n_{T}=n_{h} / 2 \Gamma$ then the fusion power $\Gamma P_{f} \Gamma$ scales as

$$
P_{f} \propto n_{D} n_{T}=\frac{n_{h}^{2}}{4}=\frac{n_{e}^{2}}{4\left(1+Z n_{z} / n_{h}\right)^{2}} \approx \frac{n_{e}^{2}}{4}\left(1-2 Z n_{z} / n_{h}\right)
$$

where $n_{e}$ and $n_{z}$ are the electron and impurity densities Trespectively. This expression thus gives the power degradation as a function of $n_{z} / n_{h}$.

For a given confinement device geometry and hydrogen core and edge parameters $\Gamma$ the impurity influx to the core is controlled by the rate of evaporation of the liquid wall. The evaporation rate is $\Gamma$ in turn $\Gamma$ determined by the wall temperature. Thus $\Gamma$ via these steps $\Gamma$ a given wall temperature results in a prediction of the core impurity level. A second major factor affecting the impurity influx is whether the divertor plate has a high or low hydrogen recycling coefficient since this impacts the impurity removal efficiency. Note that a side-wall source of impurities is considered here while impurity sputtering at the divertor plate is analyzed elsewhere at this conference [5].

The plan of the paper is as follows. The simulation model is discussed in Sec. 2. The calculated characteristics of the main hydrogen edge-plasma are presented in Sec. 3 for the low- and high-recycling regimes. The results for lithium and fluorine penetration to the core are given in Sec. 4 Tand the conclusions are listed in Sec. 5 .

\section{Geometry and Model}

The plasma edge-region is modeled as a long $\Gamma$ thin $2 \mathrm{D}$ slab corresponding to the poloidal-radial plane of a tokamak as shown in Fig. 1. A strong (toroidal) magnetic field $\Gamma B_{t} \Gamma$ is present in the third direction (into the page) $\Gamma$ with a weaker poloidal field $\Gamma$ 
$B_{p}$; the ratio is taken as $B_{p} / B_{t}=0.17$. The dimensions of the edge region are similar to the ITER device [6] $\Gamma$ and we have verified by simulations that the edge-plasma characteristics of this simplified slab model are very similar to those obtained for the actual ITER geometry.

We use the UEDGE 2D transport code to calculate the spatial distribution of the hydrogen and the impurities [7]. Equations are solved for particle continuity and parallel momentum for each ion charge-state $\Gamma$ where the parallel direction is that along the total magnetic field $\Gamma \mathbf{B}$. The inertialess parallel electron momentum equation is used to determine the parallel electric field $\Gamma E_{\|} \Gamma$ in terms of the electron pressure. Separate electron and ion temperature equations are used $\Gamma$ with all ion species assumed to have a common temperature. The hydrogen neutrals are described by a reduced Navier-Stokes model[8] $\Gamma$ and the impurity neutrals are modeled by a diffusive model (i.e. Tinertia and viscosity are neglected) with the neutral temperature fixed at $1 \mathrm{eV}$. The ionization and radiation rates for hydrogen and the impurities are taken from Refs. 9П10Гrespectively.

The parallel transport is assumed to be classical [11] with flux-limits on the viscosity thermal force Theat conductivity terms. Flux limits are also used for the hydrogen and impurity neutrals so that any diffusive flux does not exceed the product of the local thermal speed and density. The cross-field transport is assumed to be diffusive owing to plasmas turbulence with typical values from a survey of present experimental results [6]. We use coefficients for density of $D=0.33 \mathrm{~m}^{2} / \mathrm{s} \Gamma$ for electron and ion energy transport $\Gamma \chi_{e, i}=0.5 \mathrm{~m}^{2} / \mathrm{s}$; radial ion viscosity is also set to $0.5 \mathrm{~m}^{2} / \mathrm{s}$. In addition $\Gamma$ these anomalous cross-field diffusion coefficients are not allowed to exceed the Bohm rate $\Gamma D_{B}=T_{e} / 16 e B$; this is done to prevent unrealisticly large diffusion in regions near the liquid wall where $T_{e}$ may be low Tespecially when large impurity levels near the wall decrease $T_{e}$ by radiation loss. The parallel energy flux of electrons and ions to the divertor plate is set to $\delta_{e, i} n T_{e, i} c_{s}$ Twhere $\delta_{e, i}=5$ and $2.5 \Gamma$ respectively $\Gamma$ and $c_{s}$ is the ion-acoustic speed.

\section{Hydrogen Plasma Characteristics}

For liquid walls and divertors $\Gamma i t$ is possible for the divertor plasma to be in a highor low-recycling mode $\mathrm{depending}$ on the divertor material. Most materials recycle hydrogen after sustained plasma exposure leading to the high-recycling regime lithium retains the hydrogen as lithium hydride which leads to a low-recycling regime. Thus $\Gamma$ we simulate each of these regimes by using different values of the hydrogen particle-recycling coefficient $\Gamma R_{h}$ Ton the divertor plate.

For the high-recycling case $\Gamma$ we set $R_{h}=0.99 \Gamma$ the density on the core boundary $\Gamma$ 
$n_{h c}$ T to $4 \times 10^{19} \mathrm{~m}^{-3}$ Tand the power flux to $1.4 \times 10^{5} \mathrm{~W} / \mathrm{m}^{2}$ divided equally between the ions and electrons. For the low-recycling case we use the same power flux $R_{h}=0.25$ Tand $n_{h c}=2 \times 10^{19} \mathrm{~m}^{-3}$. The lower $n_{h c}$ for this second case is a qualitative recognition that the edge density will likely be lower for the low-recycling case unless very strong hydrogen edge particle-fueling is provided. Hydrogen loss is also allowed (although small here) at the outer wall where the same $R_{h}$ as at the plate is used.

The density and temperature just outside the separatrix at the midplane $(x=0)$ and the divertor plate $(x=14.5 \mathrm{~m})$ are shown in Table 1 . The low-recycling case is characterized by higher temperatures and lower densities Tespecially at the divertor plate. Another important feature of the solutions not listed in the table is that the parallel ion velocity $\Gamma v_{\| i}$ is much larger throughout the SOL for the low-recycling case. The low $v_{\| i}$ for the high recycling results from the lower $E_{\|}$Twhich is a consequence of the strong particle source from ionization of recycled particles near the plate; this reduced $E_{\|}$decreases the parallel loss of impurity ions to the plate.

Table 1.Hydrogen edge-plasma parameters for two recycling cases

\begin{tabular}{|c||c|c|c|c|}
\hline $\begin{array}{c}\text { Hydrogen } \\
\text { recycling }\end{array}$ & $\begin{array}{c}\text { Midplane } n_{e} \\
\left(10^{19} \mathrm{~m}^{-3}\right)\end{array}$ & $\begin{array}{c}\text { Midplane } T_{e} \\
(\mathrm{eV})\end{array}$ & $\begin{array}{c}\text { Plate } n_{e} \\
\left(10^{19} \mathrm{~m}^{-3}\right)\end{array}$ & $\begin{array}{c}\text { Plate } T_{e} \\
(\mathrm{eV})\end{array}$ \\
\hline \hline$R_{h}=0.25$ & 0.95 & 1090 & 0.26 & 960 \\
\hline$R_{h}=0.99$ & 3.50 & 255 & 50.9 & 76 \\
\hline
\end{tabular}

\section{Impurity Influx Calculations}

To simulate the effect of vapor from liquid walls $\Gamma$ we inject a flux of impurity neutrals uniformly along the outer wall. In chapter 8 of Ref. $2 \Gamma$ this $f\left(u x \Gamma \Gamma_{y g} \Gamma\right.$ is related to the temperature of the wall $\left(T_{w}\right)$ by fitting vapor pressure $\left(p_{v}\right)$ measurements to an Arrhenius-type relation of $p_{v}=n_{v} k T_{w} \propto \exp \left(-C / T_{w}\right)$ Tand then taking the flux to be

$$
\Gamma_{y g}=n_{v} \bar{v}_{v} / 4 \text {. }
$$

Here $n_{v}$ is the vapor density and $\bar{v}_{v}=\left(8 k T_{w} / \pi m_{v}\right)^{1 / 2}$ with $m_{v}$ the vapor mass. The resulting evaporative particle fluxes are shown in Fig. 2 for Li L SnLi (an 80\%/20\% mixture) Tand the molten salt Flibe. For Flibe $\left(\mathrm{Li}_{2} \mathrm{BeF}_{4}\right)$ Twe use only the fluorine (F ) component (which evaporates as $\mathrm{BeF}_{2}$ ) in our edge-plasma simulations for two reasons: $\mathrm{F}$ has the highest nuclear charge $Z$ C causing it to radiate most strongly $\Gamma$ and our calculations show that $\mathrm{F}$ penetrates to the core most easily of the components. Also Tbecause of momentum conservation during dissociation of the $\mathrm{BeF}_{2}$ Tone $\mathrm{F}$ atom usually be directed back to the surface; thus $\Gamma$ we take only one forward-directed $\mathrm{F}$ for each $\mathrm{BeF}_{2}$ molecule. For SnLiTthe surface layer of atoms is believed to be Li [2] $\Gamma$ with virtually no Sn evaporating; thus $\Gamma$ for the plasma modeling here we treat Li and SnLi walls the same. 
The calculated densities at the core boundary for Li and $\mathrm{F}$ versus their corresponding wall gas flux are shown in Fig. 3a for the high-recycling case and in Fig. $3 \mathrm{~b}$ for the low-recycling case. The hydrogen plasma is allowed to evolve in response to the impurities. Note that the impurity densities are larger for a given $\Gamma_{y g}$ for the high-recycling case $\Gamma$ which can be traced to the lower $E_{\|}$. The penetration of the impurities into the core is a competition between the anomalous radial $(y)$ diffusion across $\mathbf{B}$ T and the loss of the ions either back to the liquid wall or to the divertor plate which is strongly affected by the $E_{\|}$acceleration of the ions toward the plate and collisional coupling with similarly-directed hydrogen ions. It is assumed that an impurity ion has a recycling coefficient of 0.25 when it strikes the wall or the divertor plate to crudely model any self-sputtering $\Gamma$ but this effect does not contribute substantially to the core impurity density [5]. For the high-recycling case 5 the loss to the plate is small owing to the small $E_{\|}$and a comparatively large number of ions penetrate to the core-edge boundary. In contrast $\Gamma$ for low recycling $\Gamma$ the parallel loss to the plate is the dominant loss mechanism Tand the density of impurities reaching the core boundary is much reduced.

There can also be a significant different between $\mathrm{F}$ and $\mathrm{Li}$ for a given $R_{h}$. By artificially changing the properties of these ions $\Gamma$ we have identified the higher ionization potential for neutral $\mathrm{F}$ and the larger number of $\mathrm{F}$ charge states as the primary reasons for the better radial penetration of $\mathrm{F}$.

The acceptable levels of impurities at core boundary $(y=-2 \mathrm{~cm}$ here $)$ are determined from Eq. (1). Taking a $20 \%$ power degradation to define the limit $F$ can have a concentration of $\sim 1 \%$ whereas $\mathrm{Li}$ can have three times the density. Thus $\Gamma$ assuming a average core hydrogen density of $1 \times 10^{20} \mathrm{~m}^{-3}$ and a flat core impurity profile gives an allowable edge density of $\mathrm{F}$ of $1 \times 10^{18} \mathrm{~m}^{-3}$ and for Li of $3 \times 10^{18} \mathrm{~m}^{-3}$.

The upper limits of $\Gamma_{y g}$ in Figs. $3 \mathrm{aIb}$ for $\mathrm{F}$ occur with an steep rise in $n_{z c} \Gamma$ the core-boundary impurity density. These solutions are all steady-state $\mathrm{but}$ the steep rise in $n_{z c}$ is caused by $T_{e}$ at the wall dropping below 5 eV $\Gamma$ so that the $\mathrm{F}$ gas can penetrate farther radially. Since the ionization occurs farther from the wall $\Gamma$ the diffusive loss back to the wall is reduced until the density builds up. On the other hand steady-state solutions for Li are lost before the core impurity limit is reached. The non-monotonic behavior of $n_{z c}$ is caused by a Li plasma that forms just in front of the liquid wall $\Gamma$ thereby temporally improving the shielding. If $\Gamma_{y g}$ is increased beyond the end-point marked by the " $\mathrm{x}$ " in Fig. 3a for low recycling $\Gamma$ the edge $T_{e}$ profile contracts substantially in the radial direction owing to radiation from $\mathrm{Li}$ in a $T_{e}<1 \mathrm{eV}$ region where the Li radiation emissivity has a peak. This radiation/condensation or MARFE-like structure thus narrows the parallel loss channel for the Li ions Tand their density continues to build in time Teventually raising the core-edge density too high as noted by the dotted arrow. For the high-recycling case in Fig. 3b This runaway is 
delayed for some range of $\Gamma_{y g}$; the solid line shows state-state solutions $\Gamma$ and for $\Gamma_{y g}$ up to $1 \times 10^{19} \mathrm{~m}^{2} \mathrm{~s}$ Ta small $\mathrm{Ttime-dependent} \mathrm{MARFE-like} \mathrm{region} \mathrm{exists} \mathrm{near} \mathrm{the} \mathrm{plate \Gamma}$ but since most of the ion loss is already radial Tan abrupt build of Li does not occur. However $\Gamma$ for $\Gamma_{y g}$ in the range of $\sim 2 \times 10^{19} \mathrm{~m}^{2}$ s $\Gamma$ the edge density $\Gamma$ though oscillatory $\Gamma$ can exceed the dilution limit.

The results shown in Figs. 3aIb can be used to estimate the allowable temperature for lithium and Flibe walls using Eq. (2). The temperature limits are given in Table 2. We usually take the gas flux shown at the end of the curves for the maximum $\Gamma_{y g}$. From the discussion above $\mathrm{T}$ these endpoints appear to define the $\mathrm{F}$ limits well and also $\mathrm{Li}$ in the low-recycling case. The limit for $\mathrm{Li}$ in high-recycling case is somewhat hard to define precisely as discussed above.

Table 2. Impurity-based temperature limits for liquid walls

\begin{tabular}{|c||c|c|c|}
\hline $\begin{array}{c}\text { Hydrogen } \\
\text { recycling }\end{array}$ & $\begin{array}{c}\text { Lithium } \\
{ }^{\circ} \mathrm{C}\end{array}$ & $\begin{array}{c}\text { Flibe }(\mathrm{F}) \\
{ }^{\circ} \mathrm{C}\end{array}$ & $\begin{array}{c}\mathrm{Sn}_{80} \mathrm{Li}_{20} \\
{ }^{\circ} \mathrm{C}\end{array}$ \\
\hline \hline$R_{h}=0.25$ & 380 & 480 & 590 \\
\hline$R_{h}=0.99$ & $>300$ & 400 (solid) & $>500$ \\
\hline
\end{tabular}

\section{Conclusions}

We have used the 2D UEDGE transport code to predict the impurity influx into the core region of a large tokamak in the presence of liquid walls. Both high- and low-recycling hydrogen edge plasmas have been modeled for lithium and fluorine (Flibe) side walls. The impurity core-boundary densities are calculated for a range of impurity gas fluxes from the walls. Since expressions for the gas flux (or evaporation rates) of the liquids depend strongly on the wall temperature $\Gamma$ this analysis directly relates the core impurity content to the liquid wall temperature. A key issue here is the removal of the continuously injected impurities Teither by parallel loss or diffusion back to the side wall. Thus The collapse of the edge at larger Li wall injection fluxes is different than would be observed by pellet injection in the core as in TFTR [3] $\Gamma$ as we have verified by calculations using core-side injection of Li; nevertheless $\Gamma$ the results of these experiments are important to more fully understand for the low-recycling regime.

The lowest impurity influx is obtained for Li in the low-recycling mode $\mathrm{mostly}$ because this mode provides good removal of the impurities along the B-field to the absorbing divertor plate $\mathrm{Cbut}$ also because the Li radial penetration is less effective than that for F . For Flibe with $\mathrm{F}$ Tthe best case is also low-recycling. The worse case is $\mathrm{F}$ in the high-recycling mode; here the allowable temperature limit is $400^{\circ} \mathrm{C}$ Cbelow the $\sim 450^{\circ} \mathrm{C}$ melting point of Flibe; this is clearly unacceptable. Thus $\Gamma \mathrm{Li}$ Tor an SnLi 
composite Thave a significant temperature window where they can operate (Li melts just below $200^{\circ} \mathrm{C}$ ) from an impurity contamination point-of-view for a large tokamak. As discussed in Ref. 5 Tthe source of impurities from sputtering on the divertor plate does not appear to contribute significantly to core impurities. A number of issues still need to be analyzed more thoroughly 5 such as the self-consistent temperature rise of the liquid wall from radiation and neutron heating $\Gamma$ the temperature rise needed for good thermal efficiency the flow characteristics in a strong magnetic field $\Gamma$ and the effect on core MHD modes. Initial work in these areas are described in Ref. 2.

It also may be possible to improve the impurity shielding by intervention techniques. The shielding effect which reduces $n_{z c}$ for a range of gas flux for Li in Fig. 3a shows that a forming a plasma in front of the wall can be an effective attenuator of the vapor. Auxiliary heating of the $\mathrm{Li}$ or $\mathrm{F}$ vapor near the wall surface together with a pumping region near the divertor plate could be effective. A full analysis of this mechanism remains to be done.

\section{Acknowledgments}

We gratefully acknowledge useful discussions with J.N. Brooks and R.W. Moir. This work was performed under the auspices of the U.S. Department of Energy by the University of California Lawrence Livermore National Laboratory under contract No. W-7405-Eng-48. 


\section{References}

[1] R.W. Moir ГNucl. Fusion 37 (1997) 557.

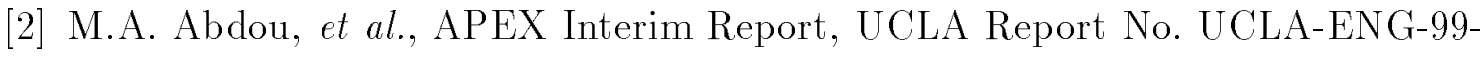
206 ГNov. Г1999.

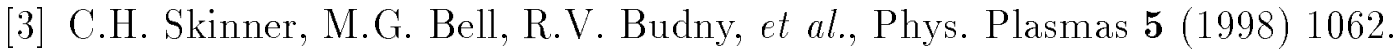

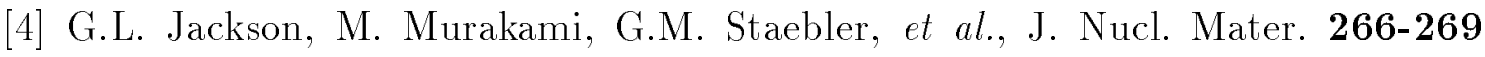
(1999) 75 .

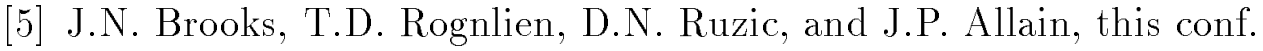

[6] ITER Physics Basis Tin Nucl. Fusion 39 (1999)ГChapt. 4Г2391.

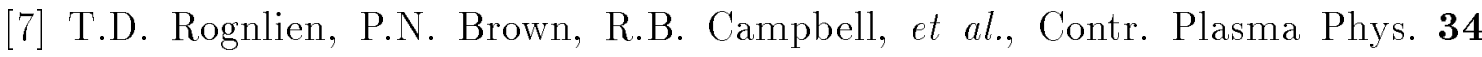

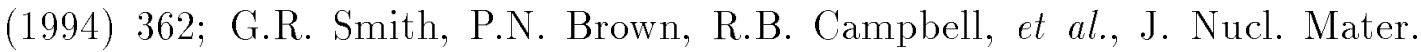
220-222 (1995) 1024.

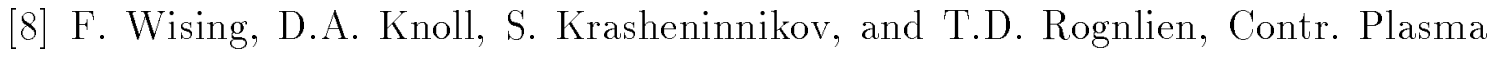
Phys.Г36 (1996)Г309.

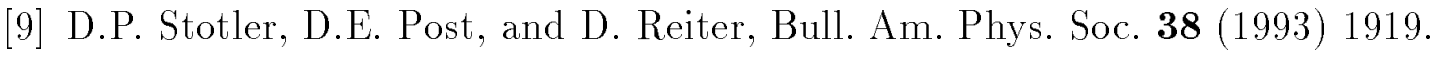

[10] R.A. HulseTNucl. Tech./Fusion 3 (1983) 259.

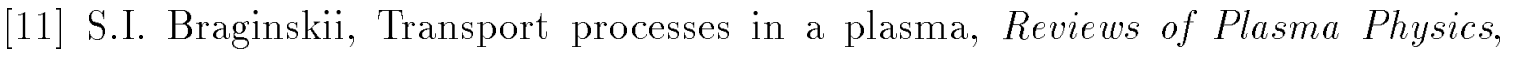
Vol. 1ГEd. M.A. Leontovich (Consultants BureauГNew YorkГ1965)Гp. 205.

\section{Figure captions}

1. The 2D geometry used to model the tokamak edge for the simulations with $x=0$ corresponding to a symmetry plane. Core-edge boundary conditions are applied at $y=-2 \mathrm{~cm}$ Tand a uniform gas flux is injected at $y=10 \mathrm{~cm}$.

2. Evaporative particle flux from various liquids versus the surface temperature. Note that Flibe is a solid below $450{ }^{\circ} \mathrm{C}$. From Chapter 8 of Ref. 2

3. Calculated impurity density at the core-edge boundary versus gas flux from the

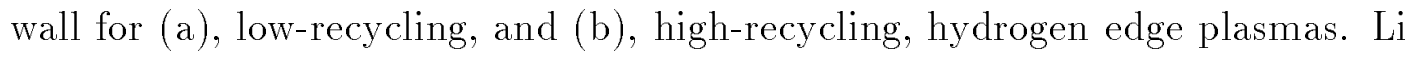
is lithium from Li or SnLi walls Tand $\mathrm{F}$ is fluorine from a Flibe wall. 


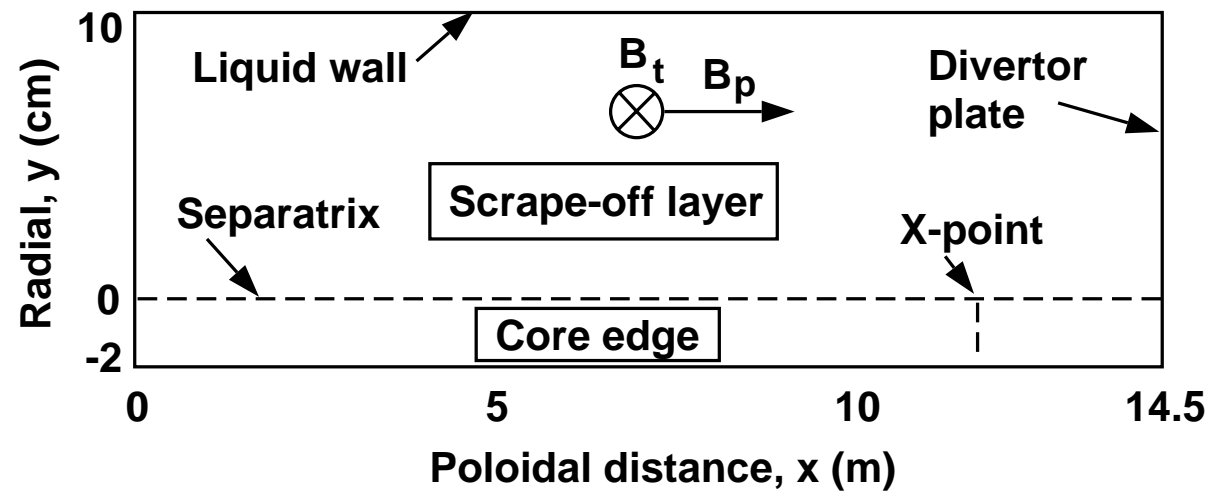

Fig. 1 


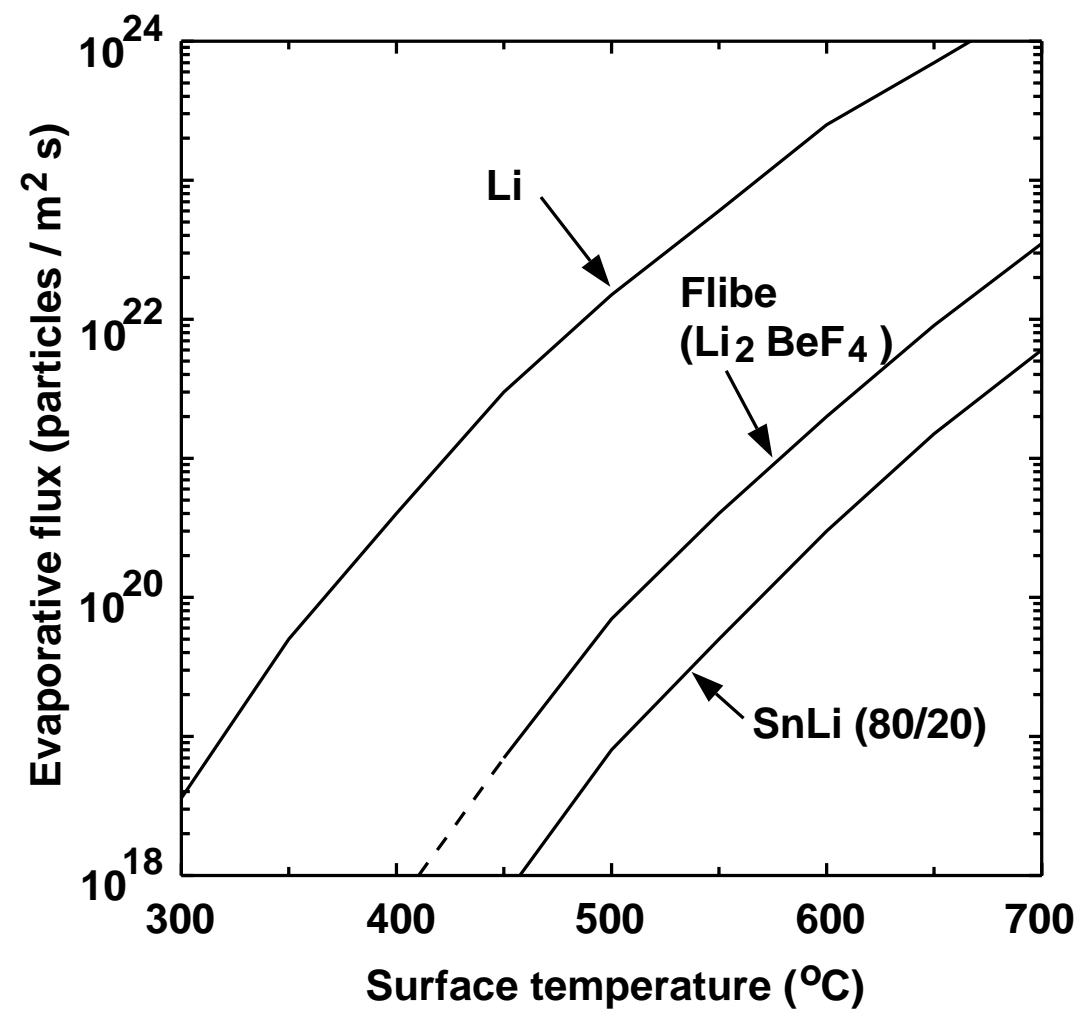

Fig. 2 

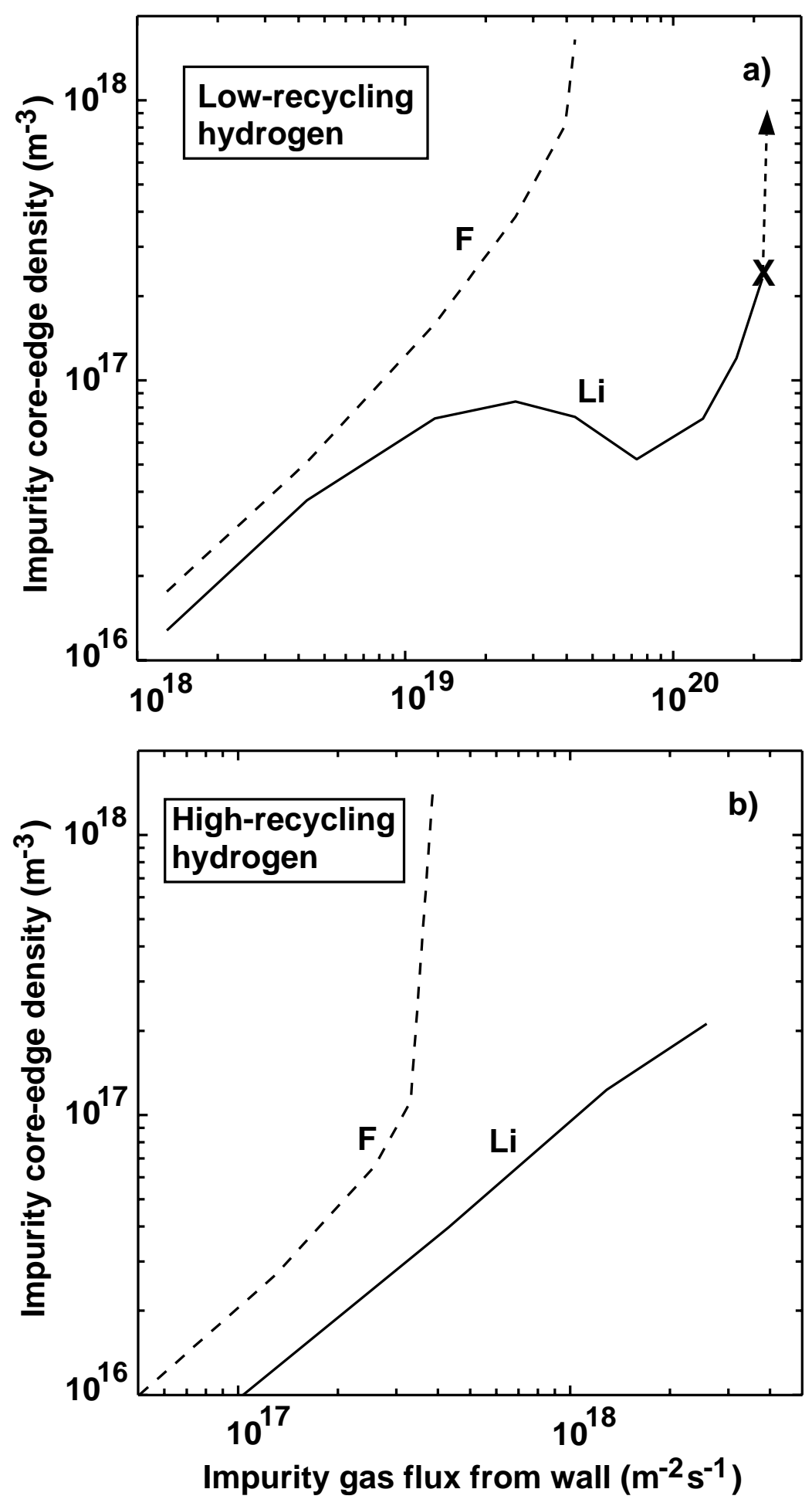

Fig. 3 